\title{
Evaluation of Confocal X-ray Analysis for Single-Atom Detection in a Thin Specimen by an Advanced Analytical Electron Microscope
}

\author{
Masashi Watanabe ${ }^{1}$ and Ray Egerton ${ }^{2}$ \\ ${ }^{1}$ Lehigh University, Bethlehem, Pennsylvania, United States, ${ }^{2}$ Department of Physics, University of \\ Alberta, Edmonton, Alberta, Canada
}

Characterization of individual atoms randomly distributed in materials, which requires atomic-scale spatial resolution as well as single-atom detection sensitivity, is one of the ultimate challenges in any chemical analysis. In fact, some of fundamental materials problems such as phase transformations and interface-related phenomena might be controlled by a few atoms within a few atomic-layers. Thus, the single-atom characterization/analysis is essential for further understanding such phenomena toward advanced materials developments. The latest aberration-corrected analytical electron microscopes (AEMs) in combination with electron energy-loss spectrometry (EELS) offers single-atom imaging and analysis [e.g. 1]. For example, it is possible to image single atoms distributed inside of a thin specimen by the confocal STEM imaging [e.g. 2]. Recently, single-atom analysis by X-ray energy-dispersive spectrometry (XEDS) has been demonstrated in aberration-corrected AEMs with the latest advance in large solid angle silicon-drift X-ray detectors (SDDs) to improve the poor signal-collection efficiency [3]. We also explored possibilities of characterization of single atoms located on surface of other support materials or membranes through the EELS and XEDS approaches [4]. Those single-atom measurements are still very challenging if these single atoms are located inside of a thin specimen. This study explores theoretically a possibility to detect single atoms inside of a thin specimen through XEDS by changing the focal point of the STEM probe, i.e. confocal XEDS analysis.

We have developed the X-ray signal simulation scheme in an oriented crystalline thin specimen by combination of electron propagation calculation with X-ray spectrum simulation [5]. For the electron propagation, called the electron wave function, the multislice approach in the XHREM code [6] was used. Then, X-ray spectra can also be calculated at each sliced layer of the wave function by porting the X-ray generation engine from legacy Desktop Spectrum Analyzer (DTSA) codes [7]. Figure 1 shows (a) [001]and (b) [010]-projected GaAs structures with single phosphorus (P) atoms located near the top surface, middle and bottom of a thin specimen. Note that $\mathrm{P}$ is a well-known impurity and usually occupied at an As site in GaAs. The wave functions of an aberration corrected probe were calculated for focus at top surface, middle and bottom for an aberration-corrected instrument operated at $200 \mathrm{kV}$ with a probe forming angle of $35 \mathrm{mrad}$ and a probe current of $250 \mathrm{pA}$ (typical operation conditions for a JEMARM200CF AEM with a cold field-emission gun and an ASCOR aberration corrector at Lehigh).

In Fig. 2, a set of simulated P K maps are summarized with respect to different focal points (top, middle and bottom) in a $22.4 \mathrm{~nm}$ thick (= 40 unit-cell thick) GaAs specimen. The single $\mathrm{P}$ atom is positioned at top, middle and bottom of the specimen, which correspond to $0,11.2$ and $22.4 \mathrm{~nm}$, (equivalent to 0,20 and 40 unit cell positions), respectively. When the $\mathrm{P}$ atom is located near the surface of the specimen, the single $\mathrm{P}$ atom is clearly detectable by adjusting the probe focus to the top surface (a typical focus position in STEM based imaging/analysis). In this probe focus condition, the $\mathrm{P}$ atom is still detectable even when the $\mathrm{P}$ atom is located at middle or bottom of this 22.4-nm-thick specimen. Conversely, the detectability of $\mathrm{P} \mathrm{K}$ signal is significantly improved by changing the probe focal point to the $\mathrm{P}$ atom positions. This simulation result implies that it is possible to obtain three-dimensional elemental distributions through the spectrometry-based techniques by applying through-focus acquisition in aberration-corrected AEMs. 


\section{GaAs with single $P$ atoms}

(a)

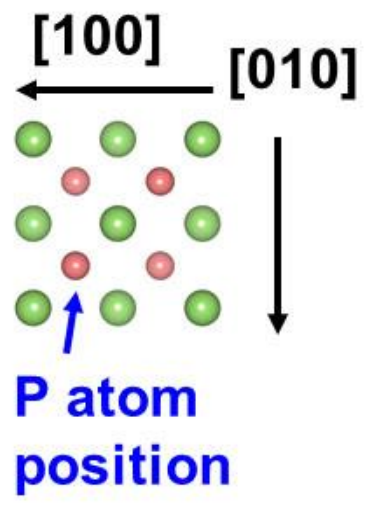

(b)
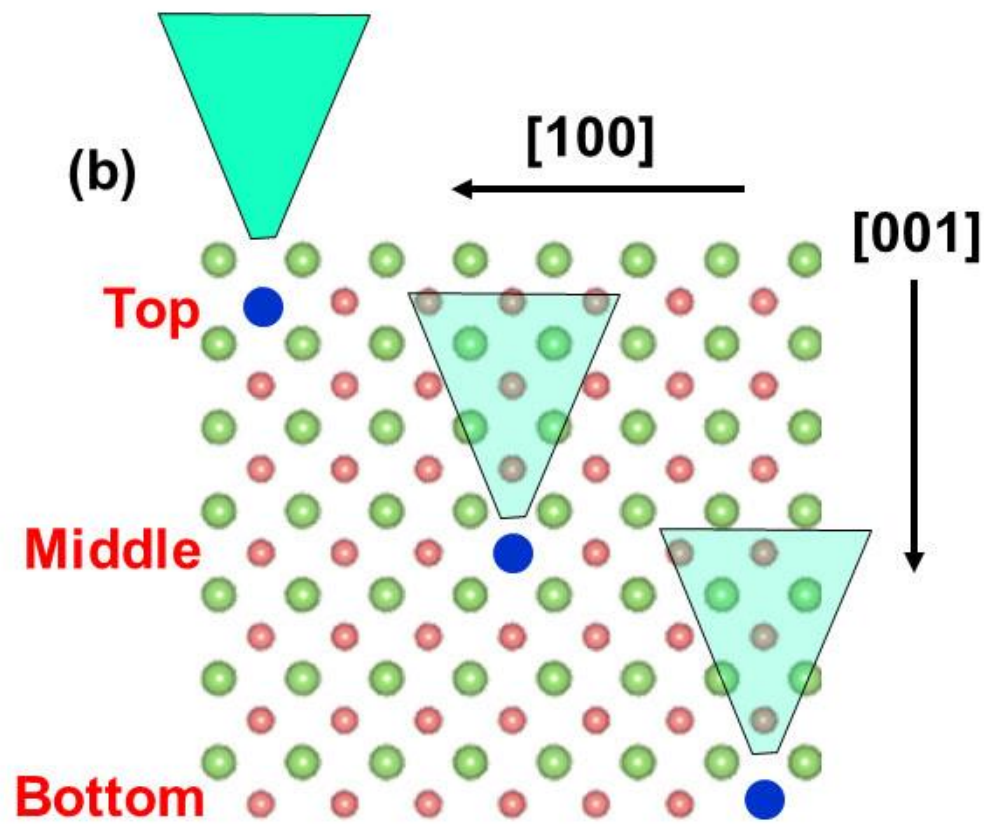

Figure 1. Schematic views of (a) [001]- and (b) [010]-projected GaAs structures, showing impurity $\mathrm{P}$ atom locations and probe focal points.

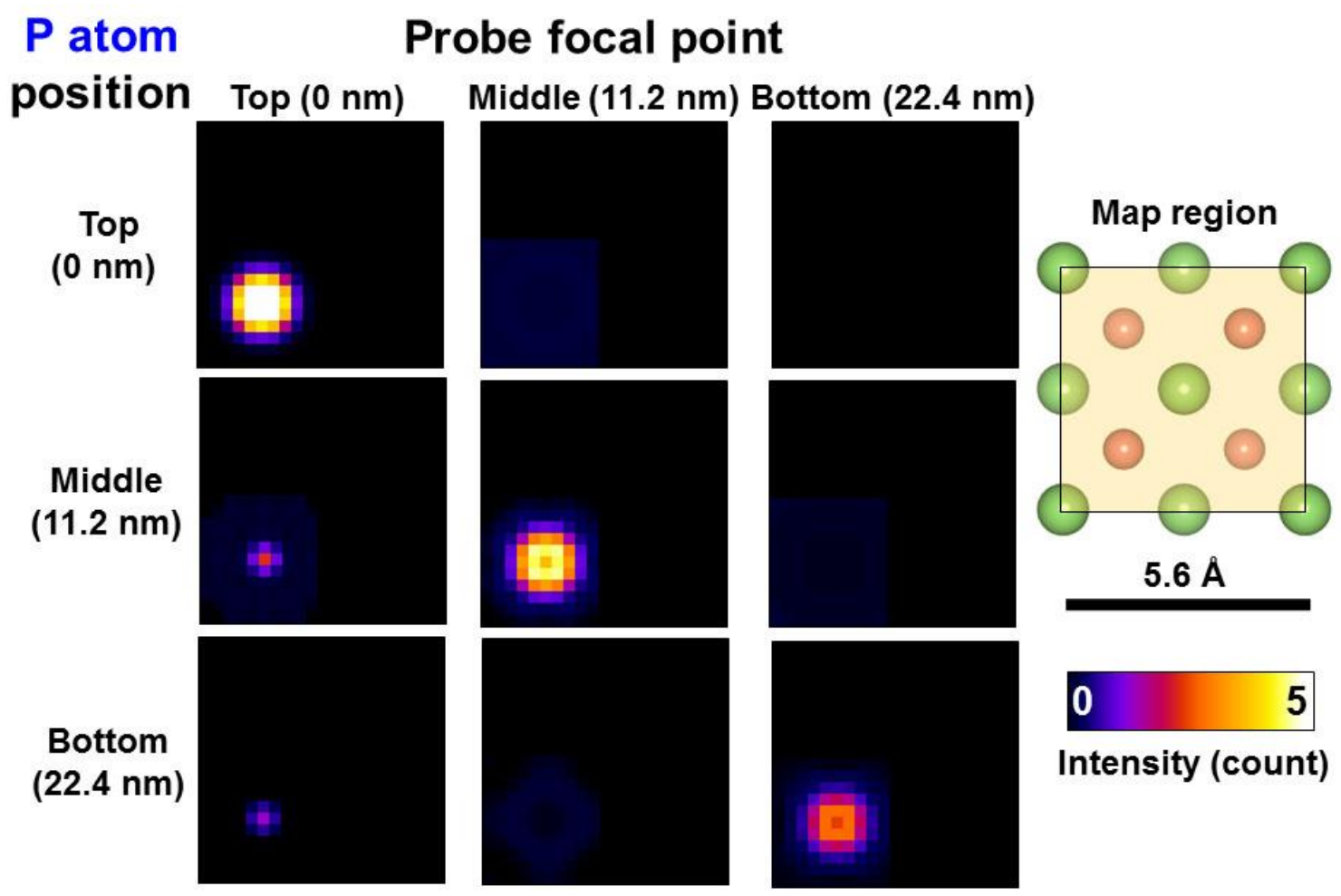

Figure 2. A set of simulated P K maps summarized as a function of the probe focal point (top, middle and bottom of the specimen) and of the $\mathrm{P}$ atom position (top, middle and bottom). 


\section{References}

[1] S.J. Pennycook \& P.D. Nellist ed. Scanning Transmission Electron Microscopy: Imaging and Analysis, Springer, NY, (2011).

[2] C.A. Wade et al Microsc. Miroana. 22 (2016), 679-689.

[3] T. Lovejoy et al., Appl.Phys. Lett. 100 (2012), 154101 (4 pages).

[4] R.F. Egerton \& M. Watanabe, Ultramicosc. 193 (2018), 111-117.

[5] M. Watanabe, Microsc. Miroana. 21 (2015), Suppl. 3, 1081-1082.

[6] K. Ishizuka., J. Electron Microsc. 50 (2012), 291.

[7] C.E. Fiori et al. Public domain DTSA software package (1992). 